\title{
Towards Smart Manufacturing with Dynamic Dataspace Alignment
}

\author{
Donatella Firmani ${ }^{1(\bowtie)}$, Francesco Leotta ${ }^{2}$, Federica Mandreoli ${ }^{3}$, \\ and Massimo Mecella ${ }^{2}$ \\ 1 Università Roma Tre, Rome, Italy \\ donatella.firmani@uniroma3.it \\ 2 Sapienza Università di Roma, Rome, Italy \\ \{leotta,mecella\}@diag.uniroma1.it \\ 3 Università di Modena e Reggio Emilia, Modena, Italy \\ federica.mandreoli@unimore.it
}

\begin{abstract}
The technological foundation of smart manufacturing consists of cyber-physical systems and the Internet-of-Things (IoT). Despite smart manufacturing has become a key paradigm to promote the integration of manufacturing processes using digital technologies, the manufacturing processes themselves are designed by human experts in a traditional way and have limited ability to adapt their behavior to exceptional circumstances. We leverage the fact that each IoT device in a smart factory can be coupled with a digital twin - that is, a software artefact that faithfully represents the physical system using real-time sensor data - to envision a software architecture to support adaptation of the manufacturing process when divergence from reference practices occur.
\end{abstract}

Keywords: Smart manufacturing $\cdot$ Digital Twins $\cdot$ Internet-of-Things

\section{Introduction}

Production processes are nowadays fragmented across different companies and organized in global multi-tier supply chains. This is the result of a first wave of globalization, fueled by the diffusion of Internet-based Information and Communication Technologies in the early years 2000. The recent wave of new Industry 4.0 technologies is further multiplying opportunities, with a wide range of services offered by small and medium-sized suppliers and automated production plants routinely employing thousands of devices from hundreds of vendors.

In such a dynamic context, smart manufacturing has become a key paradigm, using digital technologies to promote the integration of product design processes, manufacturing processes, and general collaborative business processes across factories and enterprises. Smart factories consist of a multi-layered integration of the information related to various activities along the factory and related resources. However, processes still generally reflect established work practices and have limited ability to properly adapt their behavior to exceptional circumstances where variations or divergence from reference practices occur.

(C) Springer Nature Switzerland AG 2020

S. Dupuy-Chessa and H. A. Proper (Eds.): CAiSE 2020 Workshops, LNBIP 382, pp. 53-58, 2020.

https://doi.org/10.1007/978-3-030-49165-9_5 
Example 1. Consider a cardboard manufacturing scenario where two types of die cutters are available, dubbed type 1 and type 2 , with different digital communication interfaces, potentially adopting a different language and vocabulary, and employed in two separate production processes. Suppose that a customer makes a new order for type 1 while type 1 die cutters are busy processing a former order. The cardboard manufacturer can either wait until type 1 become idle again and possibly miss its production goal or re-configure a type 2 die cutter (if available) to serve as a substitute. Such interoperability is a critical goal for the cardboard manufacturer to avoid interruptions in the production process, but traditional manual mapping and configuration practices take time and anticipating all possible scenarios at design-time is often infeasible.

In smart factories, physical devices typically have a faithful representation in the digital world, usually referred to as digital twins. A Digital Twin (DT) exposes a set of services allowing to execute certain operations and produce data describing its activity. DTs are typically used to query or manipulate the state of the shop floor and the availability of DT data can have a huge impact on the design of manufacturing processes. We can imagine shop floor data stored in a factory data space together with other information, e.g., data available from the company's employment and production history, business data and worker preferences. Such information in the data space can be used to allow agility of the manufacturing process and even automatic composition of the intermediate steps for achieving a production goal, for instance by suggesting a specific mapping for automatic configuration of the type 2 die cutter in the example above.

Our final aim is to support agile recovery of smart manufacturing processes at run-time when unanticipated events in the industrial practices occur. Our goal is to automatically detect variations with respect to reference practices and trigger the exploration of the factory data space to discover the needed services and data, thus requiring no specification of policies at design-time to handle all possible events. It is worth mentioning that the EFFRA association identifies "agile value networks" as one of the five key priorities for the Future of Factories to deliver innovative products with a high degree of personalization.

Our Contribution. In this paper we discuss available technologies that can be used to achieve our final goal and describe how a user can leverage the adaptive architecture for smart manufacturing described in [4]. The considered architecture captures indeed analogies between DTs and traditional software services (accessible for instance through Web technologies) and enables automatic integration and composition of DTs through data available in the data space.

\section{Background and Related Work}

Authors in [12] provide an overview of Industry 4.0 features in multiple reference architectures and develops a maturity model for IT architectures for data-driven manufacturing. The group around Reference Architecture Model Industry 4.0 RAMI - developed a detailed conceptual architecture and a first implementation 
of the digital twin, the open Asset Administrative Shell. RAMI still does not pursue a seamless integrated approach, which starting from processes arrives to data nor supports in-process dynamic orchestration of services and data. Authors in [3] provide a methodological and technological support to agile supply chains in the Industry 4.0 context. To this end, it sets forth an architectural framework that leverages RAMI 4.0 and addresses the methodological issue of making RAMI 4.0 capable of enabling agility in supply chains. In this paper, Business Process Management (BPM) is proposed as a mean to coordinate all the different actors of a digital factory, but no exploration policies of the data available in the factory data space are considered.

Digital Twins. Although the concept of DT dates back to 2002 [6], DTs gained popularity among researchers and practitioners only recently, with the spread of IoT technologies. Authors in [1] introduce a DT reference model for a cloudbased cyber-physical system (such as a smart city) based on a smart interaction controller using a Bayesian belief network. They provide a context-based control decision scheme that uses Bayesian networks and fuzzy logic based rules to select any of these system modes for inter-system interactions. Authors in [13] introduce a novel architecture for large-scale DT platforms including a distributed DT cooperation framework, flexible data-centric communication middleware, and the platform-based DT application to develop a reliable advanced driver assistance system. Finally, the work of [11] provides a conceptual model and specific operation mechanisms for a DT shop-floor, that is, a basic unit of manufacturing, where data from both physical and virtual sides as well as the fused data are provided to drive all the steps of the production process. For sake of completeness, we also mention the concepts of Digital Shadow (DS) and Digital Model (DM). While in DTs the state of the physical object can affect the state of the digital object and vice-versa, (i) in DSs only the state of the physical object can affect the state of the digital object, and (ii) in DMs the state of the digital object is computed via simulation. We refer the reader to [8] for more discussion.

Polystores. In digital factories, it is of strategic importance to provide effective mechanisms for searching information along diverse and distributed data sources. Data interoperability and integration techniques have been proposed as a way for resolving the structural and semantic heterogeneities that can exist between data stored in distinct repositories. However, such techniques can be difficult to implement for the many organizations deploying polyglot architectures with different data management systems [7]. Polystores [10], together with its first reference implementation BigDAWG [5] have been proposed recently as a valuable solution for this scenario. A polystore system provides a loosely coupled integration over multiple, disparate data models and query languages. In this system, queries are posed over islands of information, i.e. collections of data sources, each accessed with a single query language, and the same data can be accessed from multiple islands. The issue of data manipulation in polystores has been addressed in different papers. In [9], the authors propose the introduction of a probabilistic relation that allows the enrichment of query answering with data outside the queried data source but available in the polystore (Fig. 1). 


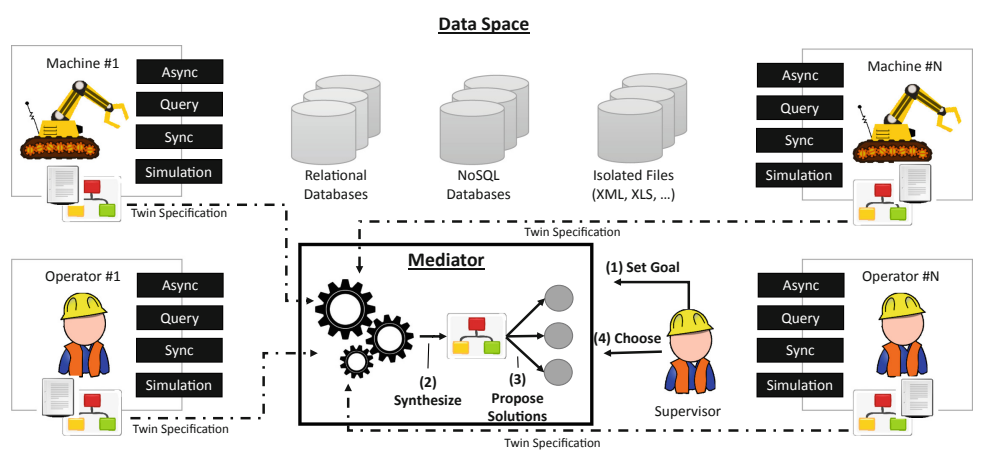

Fig. 1. The smart manufacturing architecture described in [4].

\section{Architectural Model}

We now summarize the main features of the architecture in [4] and discuss how it can be used for the purpose of agile recovery of smart manufacturing processes.

DTs wrap physical entities involved in the process. These physical entities can be manufacturing machines or human operators. A DT exposes a Web API consisting, in general, of three parts: the synchronous one, the query interface and the asynchronous one. The synchronous interface allows to give instructions to the physical entity. These instructions may, for example, produce a state change in a manufacturing machine (in case the twin is over a machine) or ask a human operator to perform a manual task (in case the twin is over a manufacturing worker). The query interface allows for asking information to the physical entity about its state and accessing the related data (possibly by applying diagnostic and prognostic functions results of machine learning). The asynchronous interface generates events available to subscribers.

The data space contains all the data available to the process. These data are heterogeneous in their nature from the access technology point of view, the employed schema (or its absence) and the employed vocabulary. It is important to note how the DTs contribute to the data space with both the query API and the asynchronous one. Other sources for the data space may include relational and no-SQL databases or unstructured sources which constitute the factory information system. In the approach in [4], the data space can be modelled as a polystore. We inherit the data modelling approach proposed in [9] where a polystore is made of a set of databases stored in a variety of data management systems, each one potentially offered by a twin through the query interface.

The human supervisor is the one defining the goals of the process in terms of both final outcomes and key performance indicators to be obtained.

The Recovery Task. Upon unanticipated events resulting in variations with respect to reference practices, available DTs and data must be integrated. This task is fulfilled by the mediator. The mediator acts in two phases: the synthesis phase and the execution/recovery phase. During the synthesis phase, the specifi- 
cations of the APIs exposed by digital twins and the meta-data (e.g. data source schemas) available in the data space, are composed in order to construct a mediator process. During the execution/recovery phase, the mediator run his program and detects possible variations with respect to reference practices (such as, the presence of a type 2 die cutter as a substitute of a type 1 die cutter in Example 1). Detection can be done by using situation calculus to model the manufacturing process, sending a run-time alert when there is no further step available in the process and identifying the DTs involved in the anomaly (such as, type 2 die cutter in Example1). Upon detection of a variation, the mediator can perform the recovery task by composing the required input/output messages requested by the anomalous DTs and thus integrating them into the regular manufacturing process. To this end, the mediator can explore the factory data space, translate and integrate the data available to comply with the format requested by the DTs involved in the recovered sequencing/interleaving. A technique for exploring data spaces modelled a polystore is described in [9]. In this context, DTs can be modeled as guarded automaton [2]: the synchronous API of the DT corresponds to input messages of the guarded automaton and the data contained in the local storage is part of the polystore defining the process.

\section{Concluding Remarks}

In this paper, we describe our preliminary effort towards an approach to automatically recover a smart manufacturing process at run-time, through automatic discovery of the needed services and data. To this end, we discussed available technologies that can be used to achieve this goal and how an adaptive architecture for smart manufacturing, based on the notion of Digital Twin (DT) can be used effectively. Our approach supports agile supply chains through innovative technological solutions aiming at the dynamic discovery of service and data flows that best fit the requirements expressed in smart manufacturing process specifications and their evolution. Next steps include developing frameworks for real manufacturing machines and company services. To this end, we are conducting a real world study in a cardboard manufacturing scenario.

Acknowledgments. This work has been partly supported by the European Commission through the H2020 project FIRST - virtual Factories: Interoperation suppoRting buSiness innovaTion (grant agreement \# 734599). The work of Francesco Leotta and Massimo Mecella has been also partly supported by the research contract with Rota Laser Dies.

\section{References}

1. Alam, K.M., El Saddik, A.: C2PS: a digital twin architecture reference model for the cloud-based cyber-physical systems. IEEE Access 5, 2050-2062 (2017)

2. Berardi, D., Calvanese, D., De Giacomo, G., Hull, R., Mecella, M.: Automatic composition of transition-based semantic web services with messaging. In: Proceedings of the 31st International Conference on Very Large Data Bases, pp. 613-624, VLDB Endowment (2005) 
3. Bicocchi, N., Cabri, G., Mandreoli, F., Mecella, M.: Dynamic digital factories for agile supply chains: an architectural approach. J. Ind. Inf. Integr. 15, 111-121 (2019)

4. Catarci, T., Firmani, D., Leotta, F., Mandreoli, F., Mecella, M., Sapio, F.: A conceptual architecture and model for smart manufacturing relying on service-based digital twins. In: 2019 IEEE International Conference on Web Services, ICWS 2019, Milan, Italy, 8-13 July 2019, pp. 229-236 (2019)

5. Duggan, J., et al.: The BigDAWG polystore system. ACM SIGMOD Rec. 44(2), 11-16 (2015)

6. Grieves, M.W.: Virtually intelligent product systems: digital and physical twins. In: Complex Systems Engineering: Theory and Practice, pp. 175-200 (2019)

7. Kadadi, A., Agrawal, R., Nyamful, C., Atiq, R.: Challenges of data integration and interoperability in big data. In: 2014 IEEE International Conference on Big Data (Big Data), pp. 38-40. IEEE (2014)

8. Kritzinger, W., Karner, M., Traar, G., Henjes, J., Sihn, W.: Digital twin in manufacturing: a categorical literature review and classification. IFAC-PapersOnLine 51(11), 1016-1022 (2018)

9. Maccioni, A., Torlone, R.: Augmented access for querying and exploring a polystore. In: Proceedings of the 34th IEEE International Conference on Data Engineering, ICDE, pp. 77-88 (2018)

10. Stonebraker, M.: The case for polystores (2015). https://wp.sigmod.org/?p=1629

11. Tao, F., Zhang, M.: Digital twin shop-floor: a new shop-floor paradigm towards smart manufacturing. IEEE Access 5, 20418-20427 (2017)

12. Weber, C., Königsberger, J., Kassner, L., Mitschang, B.: M2DDM - a maturity model for data-driven manufacturing. Procedia CIRP 63, 173-178 (2017)

13. Yun, S., Park, J.H., Kim, W.T.: Data-centric middleware based digital twin platform for dependable cyber-physical systems. In: 2017 Ninth International Conference on Ubiquitous and Future Networks (ICUFN), pp. 922-926. IEEE (2017) 